\title{
Lattice effects, stability under a high magnetic field, and magnetotransport properties of the charge-ordered mixed-valence $\mathrm{La}_{0.35} \mathrm{Ca}_{0.65} \mathrm{MnO}_{3}$ perovskite
}

\author{
M. R. Ibarra, J. M. De Teresa, J. Blasco, P. A. Algarabel, C. Marquina, J. García, and J. Stankiewicz \\ Departamento de Física de la Materia Condensada e Instituto de Ciencia de Materiales de Aragón, \\ Universidad de Zaragoza y Consejo Superior de Investigaciones Científicas, \\ Facultad de Ciencias, 50009-Zaragoza, Spain \\ C. Ritter
}

Institut Laue Langevin, B.P. 156, 38042 Grenoble Cedex 09, France

(Received 9 September 1996; revised manuscript received 14 March 1997)

\begin{abstract}
The highly $\mathrm{Mn}^{+4}$-doped compound $\mathrm{La}_{0.35} \mathrm{Ca}_{0.65} \mathrm{MnO}_{3}$ has been studied up to high temperature (700 $\mathrm{K}$ ) by using thermal-expansion, magnetostriction, magnetoresistance, and neutron-diffraction techniques. From 700 $\mathrm{K}$ down to room temperature the electrical conduction takes place through thermally activated hopping of polarons with $E_{\mathrm{hop}}=45 \mathrm{meV}$. At the charge-ordering $(\mathrm{CO})$ transition temperature $T_{\mathrm{CO}}=275 \mathrm{~K}$, pronounced anomalies in the resistivity and the lattice are observed. The neutron thermodiffractogram clearly establishes that the $\mathrm{CO}$ state occurs in the paramagnetic $(\mathrm{P})$ phase and is accompanied by a large anisotropic lattice distortion with a simultaneous large distortion of the $\mathrm{MnO}_{6}$ octahedra. The antiferromagnetic (AF) phase appears at $T_{N}=160 \pm 3 \mathrm{~K}$. At this temperature no lattice effect is observed. The CO-P and the CO-AF ground states are stable under an applied magnetic field up to 12 T. [S0163-1829(97)05437-4]
\end{abstract}

\section{INTRODUCTION}

The renewed interest in the research of mixed-valence perovskite manganese oxides due to the outstanding magnetotransport properties ${ }^{1}$ has allowed a deeper study of some properties of these compounds not discovered in the early investigations. ${ }^{2,3}$ The series $\mathrm{La}_{1-x} \mathrm{Ca}_{x} \mathrm{MnO}_{3}$ has been widely studied mainly within the ferromagnetic concentration range $0<x<0.5$, where large magnetoresistance effects are observed. The different magnetic structures were first proposed in the pioneering neutron-scattering work of Wollan and Koehler. $^{3}$ [The magnetic phase diagram has recently been established. ${ }^{4,5}$ The low-temperature phase at the rich $\mathrm{Mn}^{+4}$ hole doping $(x>0.5)$ is antiferromagnetic (AF) and insulating (I).] Wollan and Koehler ${ }^{3}$ observed a crystallographic distortion above the magnetic ordering temperature within the concentration range $0.6<x<0.75$. This was interpreted by Goodenough as an ordering of covalent bonds. ${ }^{6}$ This ordering remains below $T_{N}$ and consequently can be confirmed by analyzing the AF magnetic peaks due to the sensitivity of the diffraction spectra to the assumed distribution of the $\mathrm{Mn}^{+3}$ and $\mathrm{Mn}^{+4}$ ions at the lattice sites. ${ }^{3}$ Recently, the existence of a real-space charge ordering $(\mathrm{CO})$ in the concentration range $0.63<x<0.67$ has been inferred from electrondiffraction experiments. ${ }^{5}$ A charge-localized (CL) state was proposed to exist in $\mathrm{La}_{2 / 3} \mathrm{Ca}_{1 / 3} \mathrm{MnO}_{3}$ (Refs. 7-9) above $T_{C}$. This state was characterized by an extra anharmonic contribution over the lattice phonon one to the thermal expansion as a consequence of the lattice distortion produced by small polaron effects. A crossover from a CL to a $\mathrm{CO}$ state was proposed to occur in $\operatorname{Pr}_{2 / 3} \mathrm{Ca}_{1 / 3} \mathrm{MnO}_{3}{ }^{10}$ This was evidenced by the large magnetostriction observed as a consequence of the instability of the $\mathrm{CO}$ state under an applied magnetic field. Spontaneous and field-induced lattice effects were found to be tightly connected to the electronic state.
We report on the lattice effects and magnetotransport properties in the compound $\mathrm{La}_{0.35} \mathrm{Ca}_{0.65} \mathrm{MnO}_{3}$. For such a purpose, thermal expansion, magnetostriction, magnetoresistance, and neutron diffraction were performed up to $700 \mathrm{~K}$ in order to observe the influence of the lattice effects associated with the different ground states found in this compound and their stability under applied magnetic fields.

\section{EXPERIMENTS}

The $\mathrm{La}_{0.35} \mathrm{Ca}_{0.65} \mathrm{MnO}_{3}$ sample was prepared from stoichiometric amounts of $\mathrm{La}_{2} \mathrm{O}_{3}, \mathrm{CaCO}_{3}$, and $\mathrm{MnCO}_{3}$ by standard solid-state reaction techniques. The mixture was heated at $1000{ }^{\circ} \mathrm{C}$ overnight in air, ground, pressed bar-shaped, and heated again at $1200{ }^{\circ} \mathrm{C}$ for $24 \mathrm{~h}$, reground, repressed, and sintered at $1400{ }^{\circ} \mathrm{C}$ for $12 \mathrm{~h}$. The sample was then slowly cooled down to room temperature. Finally, it was calcined in oxygen flow at $1000{ }^{\circ} \mathrm{C}$ for $12 \mathrm{~h}$ and again, slowly cooled down to room temperature in order to achieve the adequate oxygen stoichiometry. X-ray powder diffraction (XRD) patterns were collected using a $D$-max Rigaku system with a rotating anode operating at $40 \mathrm{kV}$ and $100 \mathrm{~mA}$. The $\mathrm{Cu} K \alpha$ radiation was selected using a graphite monochromator. The XRD pattern of the $\mathrm{La}_{0.35} \mathrm{Ca}_{0.65} \mathrm{MnO}_{3}$ sample shows a single phase structure and confirms its orthorhombic symmetry that is well described by the Pbnm spatial group. The final refined unit-cell parameters at room temperature obtained by using the FULLPROF $\operatorname{program}^{11}$ are $a=5.4044(4) \AA$, $b=5.3786(4) \AA$, and $c=7.5797(5) \AA$, in agreement with previous data. ${ }^{12}$ The oxygen content was determined by redox titration using titrated potassium permanganate solution. The $\mathrm{Mn}^{4+}$ content obtained for this sample was $62 \% \pm 2$ that agrees quite well with the theoretically expected value $(65 \%)$. 


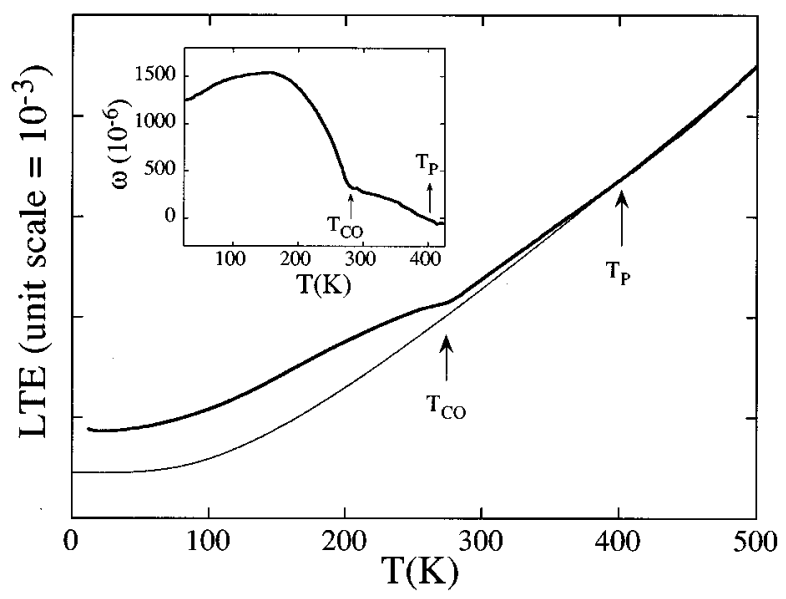

FIG. 1. Linear thermal expansion (LTE) of $\mathrm{La}_{0.35} \mathrm{Ca}_{0.65} \mathrm{MnO}_{3}$. The line is a fit of the high-temperature LTE using a Grüneisen law and a Debye temperature $\Theta_{D}=500 \mathrm{~K}$. The inset shows the volume anomaly obtained as $\omega=3\left[(\Delta L / L)_{\text {expt }}-(\Delta L / L)_{\text {cal }}\right]$ (see text).

Linear thermal expansion and magnetostriction were measured using the strain-gauge technique and the resistivity by the standard four-point technique. The magnetic field was produced by a superconducting magnet which produces steady magnetic fields up to $12 \mathrm{~T}$. The magnetoresistance was measured along the field direction and the magnetostriction parallel $\left(\lambda_{\|}\right)$and $\left(\lambda_{\perp}\right)$ perpendicular to the field. The volume magnetostriction $(\omega)$ is obtained as $\omega=\lambda_{\|}+2 \lambda_{\perp}$. The high-temperature thermal expansion was measured using a push rod and differential transformer method. Neutrondiffraction experiments were performed on powdered samples in the temperature range $1.5-320 \mathrm{~K}$ at the D1B and D2B instruments in the Institute Laue-Langevin. D1B covered the angular range $2^{\circ}<2 \theta<82^{\circ}$ with a wavelength of 2.5 $\AA$ and the high-resolution D2B up to $140^{\circ}$ and a $1.25 \AA$ wavelength.

\section{RESULTS AND DISCUSSION}

Thermal expansion measurements performed in the temperature range 4-500 $\mathrm{K}$ are displayed in Fig. 1. The hightemperature region has been fitted to a Grüneissen law considering a Debye temperature of $\Theta_{D}=500 \mathrm{~K}$. Below $T \approx 400$ $\mathrm{K}$ an extra contribution over the phonon contribution appears. In the inset of Fig. 1 we show the thermal dependence of the differential volume magnetostriction $(\omega$ $=3\left[(\Delta L / L)_{\text {expt }}-(\Delta L / L)_{\text {cal }}\right] ; \quad$ expt $=$ experimental and cal $=$ calculated). This behavior was also observed in several mixed-valence manganites ${ }^{7,8,10}$ within the paramagnetic phase. As in those cases, such behavior has been associated with the polaron traps which bring about a charge localization. At a certain temperature, called $T_{P}$ (polaronic temperature), the existence of localized carriers that distort the surrounding lattice produce an extra anharmonic contribution to the linear thermal expansion. As we will show later, the lattice polarons exist at much higher temperatures but they give anharmonic contribution to the linear thermal expansion only below $T_{P} \sim \Theta_{D}$. The polaronic regime (in this case above $T_{\mathrm{CO}}$ up to high temperatures) is dynamic in the sense that the localized electron can hop to a neighboring site, carrying the local distortion. It is still a matter of speculation if this

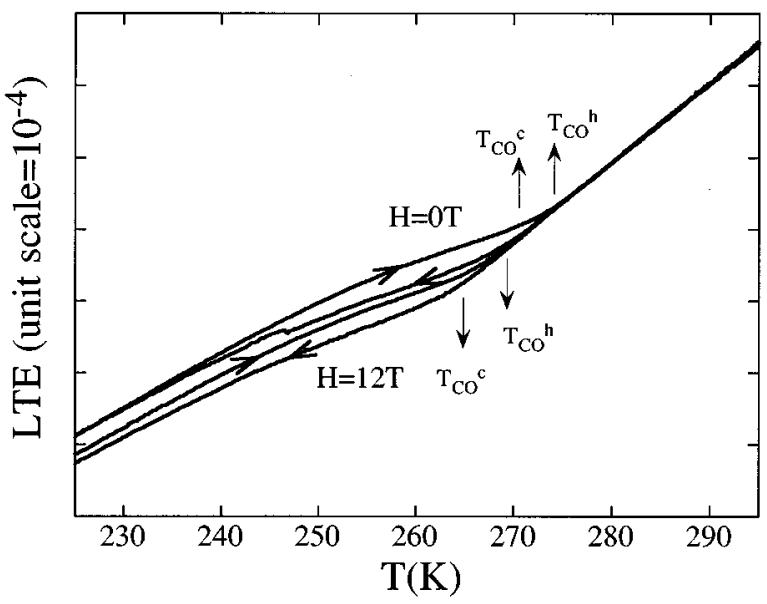

FIG. 2. Linear thermal expansion without and under an applied field of $12 \mathrm{~T}$ in $\mathrm{La}_{0.35} \mathrm{Ca}_{0.65} \mathrm{MnO}_{3}$. Arrows indicate the $\mathrm{CO}$ temperatures obtained cooling down and heating up.

anomalous $\omega$ found in the polaronic regime is related to the distortion mode of the $\mathrm{Mn}^{+3}$ octahedra (dynamic Jahn-Teller effect). The oxygen isotopic effect found in $\mathrm{La}_{0.7} \mathrm{Ca}_{0.3} \mathrm{MnO}_{3}$ (Ref. 13) supports the Jahn-Teller nature of these small polarons. A clearer anomaly is observed in the thermal expansion at $T_{\mathrm{CO}}$. As can be seen in the inset of Fig. 1, a pronounced increase of $\omega$ is observed at that temperature. We consider this lattice effect as originated by the establishment of another kind of localization process which gives rise to a static state. In this state the $e_{g}$ electrons of the $\mathrm{Mn}^{+3}$ ions are anchored at specific lattice sites making up a defined $\mathrm{Mn}^{+3}$ ( $\mathrm{Mn}^{+4}$ ) pattern, i.e., charge ordering, bringing about superlattice spots in electron-diffraction experiments. A comparison of this result with the thermal dependence of the intensity of the superlattice Bragg spots observed in electron-diffraction experiments ${ }^{5}$ clearly indicates that the anomalous volume effect observed below $T_{\mathrm{CO}}$ is related to the degree of charge ordering. As a consequence, both effects, i.e., lattice effect and charge ordering, should be ascribed to the same underlying mechanism. The charge ordering occurs in the paramagnetic $(\mathrm{P})$ phase above the long-range antiferromagnetic (AF) ordering temperature $T_{N}=160 \pm 3 \mathrm{~K}$, determined by neutron-diffraction data to be shown later. At $T_{N}$ we did not observe any significant anomaly in the thermal expansion, which indicates the absence of coupling between the lattice and magnetic behavior. In Fig. 2 we show the thermal expansion results on cooling down and warming up. A hysteretical behavior across a broad temperature interval $(\approx 75 \mathrm{~K})$ is observed, which constitutes an indication of a first-order character of the charge ordering transition.

The results of the temperature dependence of the resistivity are shown in Fig. 3. In Fig. 3(a) one can notice that the resistivity $(\rho)$ above room temperature is around $10^{-2} \Omega \mathrm{cm}$. However, at $T_{\mathrm{CO}}$ an abrupt increase in $\rho$ occurs. These measurements clearly indicate that at $T_{\mathrm{CO}}$ the mechanism of conduction changes. Analyzing the high-temperature results in detail [see inset of Fig. 3(a)], one can notice that at $T \approx 475 \mathrm{~K}$ an insulator-metal transition seems to take place. Insulatormetal transitions had been reported by Taguchi and Shimada ${ }^{14}$ in the $\mathrm{La}_{1-x} \mathrm{Ca}_{x} \mathrm{MnO}_{3}$ compounds for $0.6 \leqslant x \leqslant 0.95$ at approximately the same temperature. They proposed that a change in the electronic configuration of the 
(a)

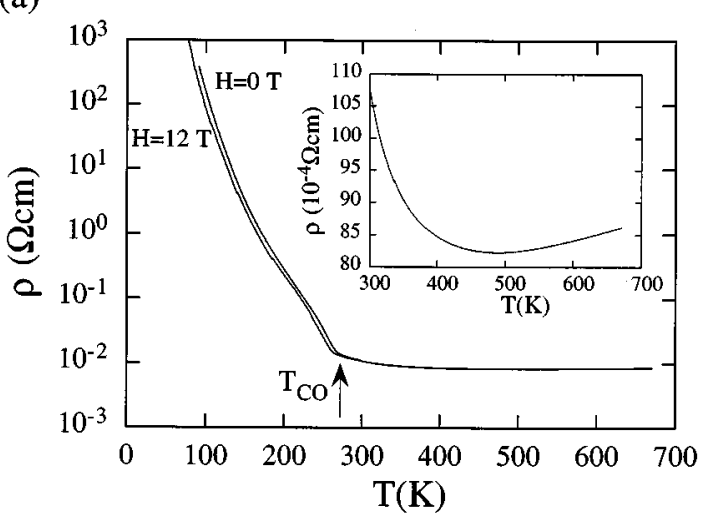

(b)

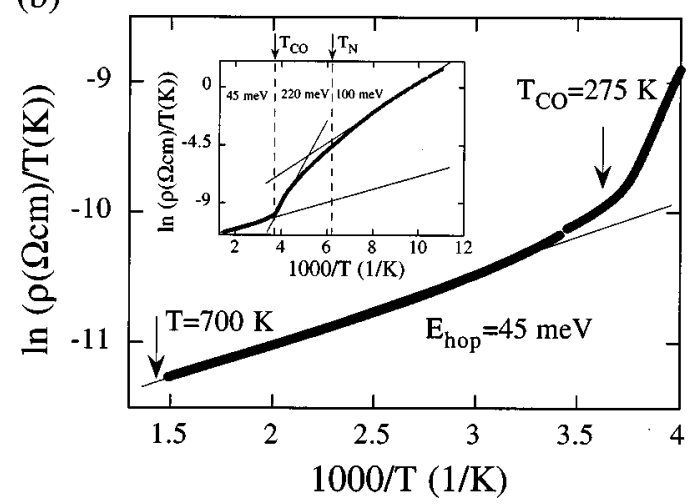

FIG. 3. (a) Thermal dependence of the electrical resistivity without and under a magnetic field of $12 \mathrm{~T}$. The inset shows in detail the high-temperature results. (b) Plot of $\ln (\rho / T)$ vs $1 / T$ across the temperature range $250-700 \mathrm{~K}$. Above room temperature the curve is linear and corresponds to polaronic conduction with a $E_{\text {hop }}$ of 45 meV (see text).

Mn ions should occur at this temperature. According to us, this is not a true metal-insulator transition but this is the expected behavior for the resistivity in a polaronic regime. The conduction in a narrow polaronic band takes place through thermally activated hopping of carriers with an activated mobility: $:^{15,16}$

$$
\rho=A T \exp \left(E_{\mathrm{hop}} / k T\right),
$$

where $A$ is a constant and $E_{\text {hop }}$ has contributions from the polaron formation energy, the transfer integral and the energy difference between identical lattice distortions with and without the hole. ${ }^{17}$ In Fig. 3(b) we have represented $\ln (\rho / T)$ vs $1 / T$. A linear behavior is obtained between 700 and 300 $\mathrm{K}$. This seems to confirm that the mechanism of conduction above room temperature is through adiabatic hopping of polarons. From the fit to Eq. (1) a value for $E_{\text {hop }}$ of $45 \mathrm{meV}$ is obtained. In $\mathrm{La}_{2 / 3} \mathrm{Ca}_{1 / 3} \mathrm{MnO}_{3}$ a value for $E_{\text {hop }} \sim 0.1 \mathrm{eV}$ was found. ${ }^{17,18}$ This indicates that, as expected, the polaronic band is strongly influenced by the $\mathrm{Mn}^{+3} / \mathrm{Mn}^{+4}$ ratio.

We have tried to extract information about the temperature dependence of the resistivity in the charge-ordering regime. However, no definite conclusion has been obtained. We show in the inset of Fig. 3(b) $\ln (\rho / T)$ vs $1 / T$ down to the lowest temperatures. There seems to exist three regimes of activation with hopping energies of $45 \mathrm{meV}$ above $T_{\mathrm{CO}}, 220$ $\mathrm{meV}$ at $T_{\mathrm{CO}}<T<T_{N}$, and $100 \mathrm{meV}$ at $T<T_{N}$. However, the

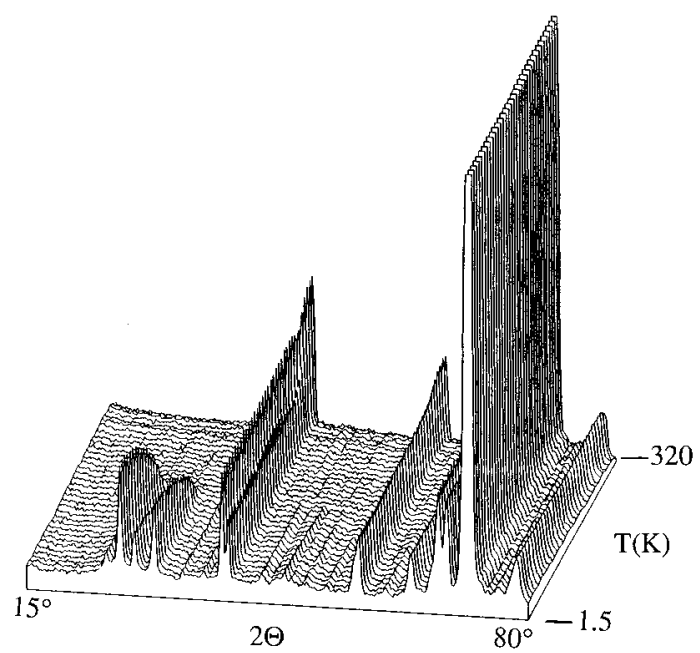

FIG. 4. Three-dimensional neutron thermodiffractogram of $\mathrm{La}_{0.35} \mathrm{Ca}_{0.65} \mathrm{MnO}_{3}$. The nuclear peak at $2 \Theta=40^{\circ}$ is split at $T_{\mathrm{CO}}=260$ $\mathrm{K}$. The AF ordering starts at $T_{N}=160 \pm 3 \mathrm{~K}$.

results for $E_{\text {hop }}$ in the charge-ordered regime could be an artefact. This can be in part because the charge-ordered state is not abruptly established within the whole sample (see the neutron results). Even in single crystals the temperature dependence of the resistivity in the charge-ordered regime seems to change from sample to sample. ${ }^{19,20}$ The resistivity below room temperature has also been measured under an applied magnetic field of $12 \mathrm{~T}$ [see Fig. 3(a)]. The only observed effect is a $5 \mathrm{~K}$ shift in $T_{\mathrm{CO}}$ towards lower temperatures. Unlike $\mathrm{Pr}_{2 / 3} \mathrm{Ca}_{1 / 3} \mathrm{MnO}_{3},{ }^{10}$ the charge-ordered state in $\mathrm{La}_{0.35} \mathrm{Ca}_{0.65} \mathrm{MnO}_{3}$ is stable under a $12 \mathrm{~T}$ magnetic field. The large magnetovolume effect found in the Pr compound associated with the suppression of the charge-ordered state is also absent in the La compound.

In Fig. 4 we show the neutron thermodiffractogram measured in the temperature range $1.5-320 \mathrm{~K}$. Below $T_{N}=160 \pm 3 \mathrm{~K}$ a set of magnetic peaks appears which coincide with the antiferromagnetic CE-type structure proposed by Wollan and Koehler. We will not go into details of the magnetic structure, which will be reported elsewhere, but on the structural aspects. A large distortion of the lattice is visible from the splitting of some of the nuclear peaks, which nevertheless preserves the orthorhombic symmetry. The nuclear peaks of the spectra were refined using the space group Pbnm starting from the same structural parameters determined from room-temperature x-ray diffraction. In Fig. 5 we show the thermal dependence of the lattice parameters. It is clearly seen the large distortion occurring at $T_{\mathrm{CO}}$. The thermal dependence of the unit-cell volume is also shown. The relative volume change $\Delta V / V$ calculated from this refinement is in good agreement with that obtained from the thermal expansion results (see Fig. 1) if we consider that the macroscopically measured strain is an average among a random distribution of grains. Under such an assumption, $\Delta V / V=3(\Delta L / L)$ and the close agreement between both measurements denote the lack of texture or preferential grain orientation in the measured polycrystalline sample.

The refinement of the neutron-diffraction results allows a determination of the average $\mathrm{Mn}-\mathrm{O} 1$ and $\mathrm{Mn}-\mathrm{O} 2$ bond lengths to be done. Here $\mathrm{O} 1$ and $\mathrm{O} 2$ stand for the apical and 


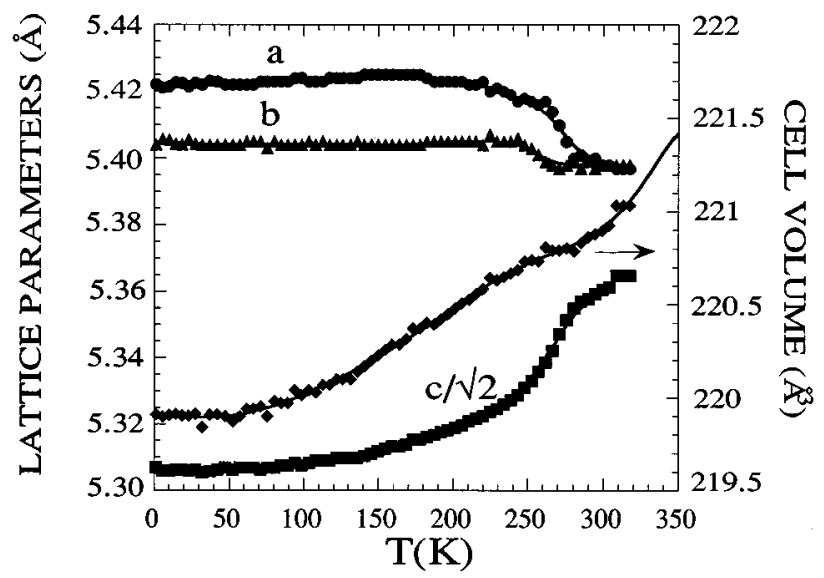

FIG. 5. Thermal dependence of the lattice parameters and unitcell volume of $\mathrm{La}_{0.35} \mathrm{Ca}_{0.65} \mathrm{MnO}_{3}$ obtained from the Rietveld refinement of the neutron-diffraction spectra.

basal plane oxygen ions, respectively. The obtained results are displayed in Fig. 6. At temperatures above $T_{\mathrm{CO}}$ the $\mathrm{MnO}_{6}$ octahedra are slightly distorted. At $T_{\mathrm{CO}}$ an abrupt change in the $\mathrm{Mn}-\mathrm{O} 2$ distances takes place, which gives rise to basalplane highly distorted octahedra. This large distortion within the basal plane observed below $T_{\mathrm{CO}}$ is consistent with Goodenough's model, ${ }^{6}$ where an orbital ordering is also predicted. It is noteworthy that data in Fig. 6 correspond to average $\mathrm{Mn}-\mathrm{O}$ distances. Consequently, as the $\mathrm{Mn}^{+4}$ octahedra are supposed to be nondistorted, the octahedra corresponding to the $\mathrm{Mn}^{+3}$ sites will be much more distorted than the average values. If we suppose that $1 / 3$ of the $\mathrm{MnO}_{6}$ are distorted, the $\mathrm{Mn}^{+3} \mathrm{O}_{6}$ octahedra will be distorted $\sim 0.2 \AA$, which is very close to the Jahn-Teller distortion of the $\mathrm{MnO}_{6}$ octahedra in $\mathrm{LaMnO}_{3}{ }^{21}$ According to the electron-diffraction measurements and the attenuation of the sound velocity at $T_{\mathrm{CO}}$, Ramirez et al. ${ }^{5}$ suggested the existence of a cooperative Jahn-Teller effect. Cooperative Jahn-Teller transitions are found in some crystalline compounds in highly concentrated systems of Jahn-Teller ions. ${ }^{22,23}$ This ordering takes place through the lattice by a strong electron-phonon interaction. One of the hallmarks of the existence of a cooperative JahnTeller transition is the softening of a phonon mode. ${ }^{24}$ From our experiment we cannot definitively conclude the JahnTeller nature of this CO transition. Nevertheless, the strong electron-phonon coupling and the simultaneous large lattice distortion and large changes in the Mn-O distances observed at $T_{\mathrm{CO}}$ would support such a model.

In this compound the magnetic behavior seems to be completely independent of the structural parameters, otherwise the magnetic ordering would occur at $T_{\mathrm{CO}}$. This is a clear indication of the weak magnetoelastic coupling in this compound between the spin system and the lattice. This weak

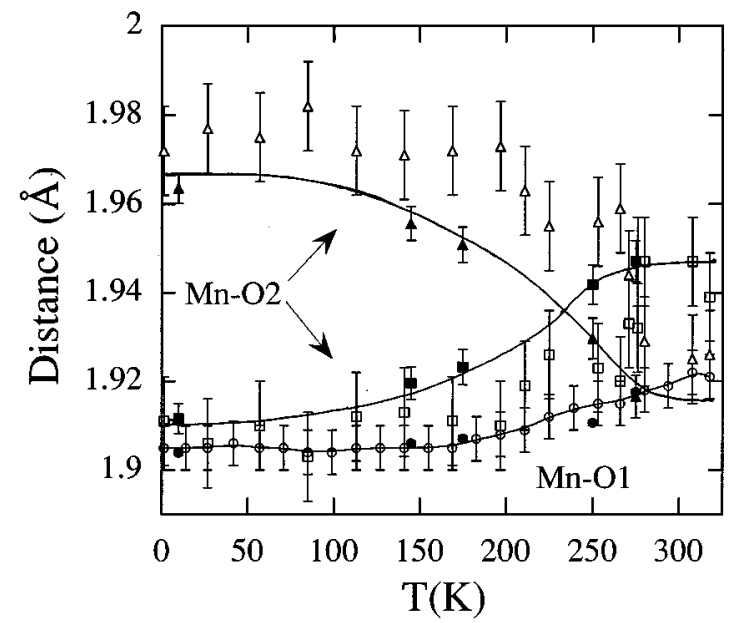

FIG. 6. Thermal dependence of the Mn-O bond lengths. O1 (circles) and $\mathrm{O} 2$ (squares and triangles) stand, respectively, for the apical and basal plane oxygen ions in the $\mathrm{MnO}_{6}$ octahedra. Open symbols are data from D1B instrument and closed symbols from D2B. Lines are visual guides. A basal plane distortion $\left(Q_{2}\right.$ mode $)$ appears below $T_{\mathrm{CO}}$.

link made the effect of the field negligible. Even at the lowest temperature and the maximum applied field, the $\mathrm{CO}$ state is stable.

\section{CONCLUSION}

From $700 \mathrm{~K}$ down to room temperature the $\mathrm{La}_{0.35} \mathrm{Ca}_{0.65} \mathrm{MnO}_{3}$ compound shows the characteristics of conduction by small lattice polarons. This is reflected in the resistivity behavior, where there is a linear relationship between $\ln (\rho / T)$ and $1 / T$ and in the linear thermal expansion, where an extra anharmonic contribution is detected. At $T_{\mathrm{CO}} \approx 275 \mathrm{~K}$, a phase transition takes place with long-range spatial ordering of carriers. In the paramagnetic $\mathrm{CO}$ state the charge ordering is accompanied by large anisotropic lattice distortions. It is worth noting, and seems to be a general rule, that the anomalous anharmonicity associated with the polaronic regime produces an isotropic volume effect. However, the establishment of the CO brings about large anisotropic lattice distortions (typical of a static Jahn-Teller effect) preserving the orthorhombic site symmetry. The antiferromagnetic CO state occurs below $T_{N} \approx 160 \mathrm{~K}$ without any change in the structural parameters. The lack of a large magnetoelastic coupling makes the ground state stable under field at any temperature and consequently the magnetoresistive and magnetostrictive effects are negligible.

\section{ACKNOWLEDGMENT}

The Spanish authors are grateful to the Spanish DGICYT for financial support under Grant Nos. APC95-123, MAT96826, and MAT96-0491.
${ }^{1}$ R. M. Kuster et al., Physica B 155, 362 (1989); R. von Helmolt et al., Phys. Rev. Lett. 63, 1990 (1993); S. Jin et al., Science 264, 413 (1994).

${ }^{2}$ G. H. Jonker and J. H. Van Santen, Physica (Amsterdam) 16, 337 (1950); 16, 599 (1950).

${ }^{3}$ E. O. Wollan and W. C. Koehler, Phys. Rev. 100, 545 (1955).
${ }^{4}$ P. Schiffer, A. P. Ramirez, W. Bao, and S.-W. Cheong, Phys. Rev. Lett. 75, 3336 (1995).

${ }^{5}$ A. P. Ramirez, P. Schiffer, S.-W. Cheong, C. H. Chen, W. Bao, T. T. M. Palstra, P. L. Gammel, D. J. Bishop, and B. Zegarski, Phys. Rev. Lett. 76, 3188 (1996).

${ }^{6}$ J. B. Goodenough, Phys. Rev. 100, 564 (1955). 
${ }^{7}$ M. R. Ibarra, P. A. Algarabel, C. Marquina, J. Blasco, and J. García, Phys. Rev. Lett. 75, 3541 (1995).

${ }^{8}$ J. M. De Teresa, M. R. Ibarra, J. Blasco, J. García, C. Marquina, P. A. Algarabel, Z. Arnold, K. Kamenev, C. Ritter, and R. von Helmolt, Phys. Rev. B 54, 1187 (1996).

${ }^{9}$ P. G. Radaelli, M. Marezio, H. Y. Hwang, S.-W. Cheong, and B. Batlogg, Phys. Rev. B 55, 3015 (1977).

${ }^{10}$ J. M. De Teresa, M. R. Ibarra, P. A. Algarabel, C. Marquina, and S. Oseroff, Phys. Rev. B 54, R12 689 (1996).

${ }^{11}$ J. Rodriguez-Carvajal, Physica B 192, 55 (1993).

${ }^{12}$ H. Taguchi and M. Shimada, J. Solid State Chem. 67, 37 (1987).

${ }^{13}$ G.-M. Zhao, K. Conder, H. Keller, and K. A. Muller, Nature (London) 381, 676 (1996).

${ }^{14}$ H. Taguchi and M. Shimada, J. Solid State Chem. 63, 290 (1986).

${ }^{15}$ D. Emin and T. Holstein, Ann. Phys. (N.Y.) 53, 439 (1969).

${ }^{16}$ Gerald F. Dionne, J. Appl. Phys. 79, 5172 (1996).
${ }^{17}$ M. Jaime et al., Phys. Rev. B 54, 11914 (1996).

${ }^{18}$ D. C. Worledge et al., J. Appl. Phys. 80, 5158 (1996).

${ }^{19}$ Y. Tomioka, A. Asamitsu, Y. Moritomo, H. Kuwahara, and Y. Tokura, Phys. Rev. Lett. 74, 5108 (1995).

${ }^{20}$ Y. Tokura, H. Kuwahara, Y. Moritomo, Y. Tomioka, and A. Asamitsu, Phys. Rev. Lett. 76, 3184 (1996).

${ }^{21}$ S. Sapathy, Z. S. Popovic, and Filip R. Vukajlovic, Phys. Rev. Lett. 76, 960 (1996).

${ }^{22}$ Z. Jirak, S. Krupicka, Z. Simsa, M. Dlouha, and S. Vratislav, J. Magn. Magn. Mater. 53, 153 (1985).

${ }^{23}$ S. Gota, J. García, J. Chaboy, and J. Bartolomé, Phys. Rev. B 44, 11632 (1991).

${ }^{24}$ B. Lüthi and W. Rehwald, in Structural Phase Transitions I, Topics in Current Physics Vol. 23, edited by K. A. Müller and H. Thomas (Springer-Verlag, Berlin, 1981), p. 131. 